\title{
Multistep Wind Speed Forecasting Based on a Hybrid Model of VMD and Nonlinear Autoregressive Neural Network
}

\author{
Yuqiao Zheng $\mathbb{D}^{1},{ }^{1}$ Bo Dong, ${ }^{1}$ Yuhan Liu, ${ }^{1}$ Xiaolei Tong, ${ }^{1}$ and Lei Wang ${ }^{2}$ \\ ${ }^{1}$ School of Mechanical and Electronical Engineering, Lanzhou University of Technology, Lanzhou 730050, China \\ ${ }^{2}$ College of Automation, Chongqing University, Chongqing, China \\ Correspondence should be addressed to Yuqiao Zheng; zhengyuqiaolut@163.com
}

Received 9 November 2020; Revised 11 December 2020; Accepted 21 December 2020; Published 22 January 2021

Academic Editor: Heng Liu

Copyright (C) 2021 Yuqiao Zheng et al. This is an open access article distributed under the Creative Commons Attribution License, which permits unrestricted use, distribution, and reproduction in any medium, provided the original work is properly cited.

Reducing the costs of wind power requires reasonable wind farm operation and maintenance strategies, and then to develop these strategies, the 24-hour ahead forecasting of wind speed is necessary. However, existing prediction work is mostly limited to 5 hours. This work developed a diurnal forecasting methodology for the regional wind farm according to real-life data of the supervisory control and data acquisition (SCADA) system of a wind farm from Jiangxi Province. The methodology used the variational mode decomposition (VMD) to extract wind characteristics, and then, the characteristics were put in the nonlinear autoregressive neural network (Narnet) and long short-term memory network (LSTM) for prediction; the forecast results of VMD-Narnet and VMD-LSTM are compared with the actual wind speed. The comparison results indicate that compared with the LSTM, the Narnet improves the accuracy by $61.90 \%$ in 24 hours on wind speed forecasting, and the predicted time horizon was improved by 6.8 hours. This work strongly supports the development of wind farm operation and maintenance strategies and provides a foundation for the reduction of wind power costs.

\section{Introduction}

The wind has attracted considerable attention as cleaning, renewable energy. Over the past decades, the installed capacity of wind power has increased dramatically and will continue to increase in the future. As the Global Wind Energy Council (GWEC) reported, the global wind industry has increased more than $50 \mathrm{GW}$ of new capacity each year since 2014 and expected $55 \mathrm{GW}$ to be increased each year until 2023 [1]; however, the price of electricity from wind was higher than that from coal-fired power, and the latter was the dominating source of electricity supply due to price reasons. Thus, how to achieve cheaper electricity out of the wind has become increasingly prominent. To reduce wind power costs, beneficial wind farm operations and maintenance strategies that are derived from diurnal wind speed forecasting are essential. Accurate diurnal wind speed forecast decreases wind curtailment, limiting electricity effectively, utilizes wind energy further, and then provides beneficial information on wind power consumption or reduction of maintenance time and reduces the cost of power generation [2]. Therefore, accurate diurnal wind speed forecasting is particularly noteworthy for wind farms.

The literature survey suggests that few studies mentioned the long-term (over 24 hours) prediction of wind speed, and the results of these studies are achieved by splitting longterm predictions into continuous short-term predictions $[3,4]$; such approaches are short-term forecasting essentially. It is reported that the result of 24-hour ahead forecasting is the most point for the power company [5]. Tian et al. also supported that wind speed prediction research should take into account the actual demand of wind farms, and they developed an Elman-based prediction system [6]. However, they still did not mention daily wind speed prediction. Subsequently, researchers have proposed different referable methods in short-term wind speed forecasting, and it has been proved that machine learning and deep learning algorithms are effective forecasting approaches [7, 8]. Han et al. studied the application effect of statistical model and neural network algorithm by comparing three different 
methods on the 1-hour wind speed forecasting, and the conclusion is that compared to statistical models, the neural network has superiority in the treatment of nonlinear characteristics of wind speed, which verified the effectiveness of the neural network in wind speed forecasting issue. Meanwhile, the parameter adjustment of the neural network that considered the autocorrelation analysis of wind made the forecasting results more accurate [9], and the study of Shi et al. also demonstrated similar conclusions on autocorrelation analysis of wind [10]. Furthermore, the gate recurrent unit (GRU) [11,12] and long short-term memory network (LSTM) that have the time correlation are considered in wind speed forecasting issue fully. Li et al. compared the performance of ANN and GRU models in wind speed prediction and indicated that the error (mean absolute error, MAE) of forecasting by GRU was less than the counterpart of ANN [13]. It testified to the algorithm considering the time correlation of wind has improved accuracies of prediction. Especially, Liu et al. showed that the LSTM and variational mode decomposition (VMD) hybrid method can accurately forecast wind speed within 1 hour [14].

Although the combination of autocorrelation and neural networks brought to more accurate wind speed prediction, wind speed forecasting still faced the challenge on the nonlinear characteristics of wind. Most researchers have considered signal decomposition algorithms to preprocess such characteristics. Liu et al. attempted to improve the wind speed forecasting accuracy of intelligent algorithms by compositing methods twice (wavelet packet decomposition and complete ensemble empirical mode decomposition), the most accurate result of the prediction had an error (mean absolute percentage error, MAPE) of $4.82 \%$ in 1 hour [15], and the conclusions of the study strongly prove the validity of the signal decomposition algorithm in wind speed prediction. Besides, Zhang et al. verified that VMD improves the accuracy of wind speed prediction by a GA-based backpropagation neural network on one step [16]. Zhang et al. also processed wind speed data for VMD, wavelet decomposition, and principal component analysis and adopted a neural network algorithm to predict wind speed [17]. The combination of signal decomposition and neural network considering the time correlation of wind is considerable according to these studies. Furthermore, there are other studies on wind speed also. Tian et al. used chaos theory to describe changes in wind speed along the timeline [18], and they observed chaotic attractors through phase space reconstruction and proposed that large prediction steps would reduce the accuracy of wind speed prediction. Yuan et al. suggested that the predictable duration of wind speed is not stable [19], which means that any prediction of wind speed requires a judgment about the predictability of wind speed.

Besides, the inconsistent data set of prediction of studies above is confusing. The wind speed data observed by meteorological observation stations are utilized to study meteorological law normally, and it is difficult to make contributions to specific wind turbines. It is suggested using wind speed data collected by the supervisory control and data acquisition (SCADA) system instead [20], which has consistent sampling interval, makes access easier, and is more indispensable in wind farm operational problems than the data from the meteorological observation station.

For the requirement of cost reduction of wind farms, this work proposed a hybrid model of the VMD and the nonlinear autoregressive neural network (Narnet) on diurnal wind speed forecasting and compared the prediction of VMD-Narnet with the counterpart of VMDLSTM. Concretely, the decomposition layer of VMD was determined by observing the central frequency and the ratio of the adjacent central frequency of intrinsic mode function (IMF) components, and we applied the VMD method to extract the IMF for processing the nonstationary and nonlinear characteristics. As IMF component inputs of the Narnet and LSTM, we predicted wind speed 24-hour ahead, then compared the prediction results of the two models, analyzed the prediction step size of the Narnet in 24 hours, computed MAE and MAPE to evaluate prediction results, and completed daily wind speed prediction mission.

In detail, the main contributions of our work are as follows:

(1) A system framework for daily wind speed prediction based on SCADA data is proposed, which ensures that each wind turbine has its wind speed prediction and the targeted operation and maintenance of each wind turbine.

(2) The induction of a novel hybrid model combined VMD, Narnet, and sample entropy for each wind turbine in farms. It improved the accuracy of medium-term wind speed forecasting and will provide a new judgment source for operation and maintenance of the turbine.

(3) Validation of the neural network with time variable can better predict wind speed, and the Narnet model has a prediction accuracy of $61.9 \%$ higher than the LSTM under the same input.

\section{Hybrid Wind Speed Prediction Model}

2.1. Variational Modal Decomposition. Although the effects of empirical mode decomposition (EMD) are widespread, the signal decomposition process still lacks mathematical theory to explain, which is the advantage of the VMD. The signal decomposition is built for the variational constraint model by VMD, and then, the introduction of the Lagrange multiplier transforms the constraint model to a nonconstrained variational problem. In this work, we set $\left\{u_{k}\right\}$ as IMF of wind speed data, $\left\{\omega_{k}\right\}$ as the central frequency of IMF, and $k$ as the quantity of IMF components.

The unconstrained variational problem of wind speed decomposition is defined as follows [21]: 


$$
L\left(\left\{u_{k}\right\},\left\{\omega_{k}\right\}, \lambda\right)=\alpha \sum_{k=1}^{k}\left\|\partial_{t}\left[\left(\delta(t)+\frac{j}{\pi t}\right) * u_{k}(t)\right] e^{-j \omega k t}\right\|_{2}^{2}+\left\|f(t)-\sum_{k=1}^{k} u_{k}(t)\right\|_{2}^{2}+\left\langle\lambda(t), f(t)-\sum_{k=1}^{k} u_{k}(t)\right\rangle .
$$

With the $n$ iterations, $\left\{u_{k}\right\}$ and $\left\{\omega_{k}\right\}$ were calculated as

$$
\left\{\begin{array}{l}
\widehat{u}_{k}^{n+1}(\omega)=\frac{\widehat{f}(\omega)-\sum_{i \neq k} \widehat{u}_{i}(\omega)+(\widehat{u}(\omega) / 2)}{1+2 \alpha\left(\omega-\omega_{k}\right)^{2}}, \\
\omega_{k}^{n+1}=\frac{\int_{0}^{\infty} \omega\left|\widehat{u}_{k}(\omega)\right|^{2} \mathrm{~d} \omega}{\int_{0}^{\infty}\left|\widehat{u}_{k}(\omega)\right|^{2} \mathrm{~d} \omega} .
\end{array}\right.
$$

In equations (1) and (2), $L($.$) is the nonconstrained$ variational problem; $\lambda(t)$ is the introduced Lagrangian multiplier; $\alpha$ is the introduced penalty factor; $\delta(t)$ is the Dirac function; $e^{-j \omega k t}$ is a phasor describing the rotation of the complex signal in time; $f(t)$ is the observed signal of wind speed; $\widehat{u}_{k}^{n+1}, \widehat{u}(\omega)$, and $\widehat{f}(\omega)$ is the Fourier transform of $u_{k}^{n+1}, u(\omega)$, and $f(\omega)$, respectively; and "** denotes the convolution operation. The VMD needs to specify the number $k$ of IMFs firstly and then the unilateral spectrum of each IMF obtained by Hilbert transform and determines the spectrum "baseband" of each IMF by exponential mixing tuning to the corresponding estimated center frequency; ultimately, the estimated bandwidth is obtained by the square norm of the gradient [21]. The $k$ value can be determined by combining the central frequency observation method and the frequency ratio of adjacent centers [22]. When the central frequency $\left(\omega_{k}\right)$ of each IMF component remains stable under different $k$ values, it shows that there is no overdecomposition of the original wind speed data. When the frequency ratio of adjacent centers is greater than $90 \%$, it indicates that the primal wind speed data are overdecomposed, and there are false components obtained from overdecomposition in the IMF components. To be specific, it is suggested that the decomposition of the IMF is stable if the amplitude of the central frequency is less than 100 for the same IMF under different $k$, and then, the decomposition of the wind speed signal is complete if all IMFs are stable. For example, if all IMFs are stable when the $k$ is not less than 18, the decomposition of the signal is complete when the $k$ is not less than 18 . Furthermore, it is necessary to make $k$ greater than 18 to decompose the signal and compute the central frequency ratios of different IMFs, and the decomposition is excessive if the central frequency of adjacent IMFs is greater than $90 \%$ under the same $k$, like 19 ; thus, the $k$ is 18 .

2.2. Nonlinear Autoregressive Neural Network. The Narnet is a shallow artificial neural network considering time delay, which effectively identifies the nonlinear mode of IMF components of the wind speed. Additionally, the autocorrelation property of the wind speed in the time axis is taken into account in the calculation of the time-delay variable [23]. In this work, we adopt the Levenberg-Marquardt algorithm on the backpropagation of the Narnet model. Let $y$ be the IMF component value of the actual wind speed, and the Narnet network neuron input function expressed is given by the following equation:

$$
z(y)=\sum_{i=0}^{d} w_{i}^{\prime} y_{t-i}+b,
$$

where $d$ is the time-delay number, $y_{t}$ is the IMF component value of the actual measured wind speed at moment $t, \hat{y}_{t}$ is the IMF component value of the predicted network wind speed at moment $t, w_{i}$ is the hidden layer weight, and $b$ is the bias. The neuron activation function selects the hyperbolic tangent function:

$$
a(z)=\frac{\left(e^{z}-e^{-z}\right)}{\left(e^{z}+e^{-z}\right)},
$$

where $z$ is the input function value of the neuron and $a$ is the activation function of the neuron. The activation function of neurons in the output layer is the linear transfer function:

$$
f(a)=\sum_{j=1}^{n} a_{j} .
$$

In equation (5), $f$ is the linear transfer function and $a$ is the value of the neuronal activation function. The multistep prediction of the IMF component of wind speed was obtained by the iteration $\hat{y}$ of the Narnet model, and the linear superposition of the IMF component of wind speed was the result of the prediction. The topology structure of the Narnet network model is constructed through equations (3)-(5), as shown in Figure 1.

2.3. Long Short-Term Memory Network. LSTM is a recurrent neural network variant model, whose deep network structure can learn the nonlinear model of IMF component of wind speed, and the threshold structure in neurons enables LSTM to have time-delay information transmission capability [24], which can deal with the time-dependent 
relationship of wind speed. Here, the backpropagation of the LSTM model adopts the Adam algorithm.

Let $y_{t}$ be the IMF component value of current input wind speed, $\hat{y}_{t}$ be the IMF component value of current forecast wind speed, and $c_{t}$ be the threshold state of neurons.

The neuronal transfer function of the LSTM network selects the hyperbolic tangent function (as shown in equation (4)), and the threshold state transfer function is the sigmoid function [25].

The multistep predicted value of the IMF component of wind speed was obtained by the $\hat{y}$ iteration of the IMF component of the LSTM model, and the linear superposition of IMF component was the predicted value of wind speed. The constructed model network topology is shown in Figure 2.

In Figure 2, $y_{t}$ is the IMF component value of wind speed at moment $t, \hat{y}_{t}$ is the IMF component value of the current forecast wind speed, $z_{f}$ is the forgetting gate input (as shown in equation (3)), $f_{t}$ is the forgetting gate state at moment $t, z_{i}$ is the input gate input, $z_{c}$ is the current unit state input, $i_{t} * \widetilde{c}_{t}$ is the input gate state at moment $t, z_{o}$ is the output gate input, $o_{t}$ is the output gate state at moment $t, c_{t}$ is the unit state, $[\cdot, \cdot]$ represents the vector splicing operation, ${ }^{*}$ represents multiplication by vector elements, $\sigma$ is the sigmoid function, and $\tan h$ is the hyperbolic tangent function.

2.4. Evaluation Index. The MAE and MAPE were utilized as quantitative evaluation indexes for the prediction of the hybrid intelligent algorithm on wind speed forecasting.

Set $y=\left\{y_{1}, y_{2}, \ldots y_{n}\right\}$ as the observed wind speed value and $\hat{y}=\left\{\hat{y}_{1}, \hat{y}_{2}, \ldots \hat{y}_{n}\right\}$ as the model predicted wind speed value. MAE and MAPE are calculated as follows:

$$
\begin{aligned}
\text { MAE } & =\frac{\sum_{1}^{n}\left|y_{t}-\hat{y}_{t}\right|}{n}, \\
\text { MAPE } & =\frac{\sum_{1}^{n}\left|\left(y_{t}-\widehat{y}_{t}\right) / y_{t}\right|}{n} .
\end{aligned}
$$

In equations (6) and (7), $n$ is the predicted step size and $t$ is the current predicted step size.

\section{Case Study}

According to the contents mentioned above, we determined the $k$ of IMF and decomposed the collected wind speed data by VMD firstly; then, we put the IMF components in the prediction model (Narnet and LSTM), respectively, to obtain the estimated values of each IMF. Furthermore, to achieve the 24-hour ahead predictions, we regard the one step-ahead prediction as a new input of the trained model and obtain the next one-step prediction. The iteration of prediction in the trained model proved the 24 -ahead predictions finally.

Here, the wind speed data were recorded by the SCADA system of a wind farm in Jiangxi Province, and data set from July 2018 to July 22, 2019, were recorded every $10 \mathrm{~min}$. The data contain 54,555 measured values, and there are missing and repeating in it. Firstly, the repeated values are deleted, and the wind speed data of 387 days are divided into diurnal sets according to the natural day. Then, the diurnal set which is less than 24 hours is deleted. Ultimately, we acquired 342day sets of wind speed. To avoid the illogic problem on time, that is, using later wind speed data to predict past wind speed data, the prediction target is the wind speed of the last day in this study ( $342^{\text {nd }}$ day).

By the variational modal decomposition strategy described before (see equation (1)), variational modal decomposition is performed for wind speed data on days 1 to 341. The preset number $k$ of IMF component of wind speed data is determined by the central frequency observation method combined with the frequency ratio of adjacent centers. The frequencies of IMF centers corresponding to different $k$ values are listed in Table 1 .

Table 1 shows that the IMF component's central frequency $\left(\omega_{k}\right)$ of each wind speed tends to be stable. The adjacent central frequency ratios of each wind speed IMF were calculated, and it was found that when there were IMF components (IMF 18, IMF 19, IMF 20, and IMF 21) with a central frequency ratio greater than $90 \%$, there were false components in the IMF component of decomposed wind speed, and the IMF component of wind speed $k$ was less than 19. Therefore, the number of IMF wind speed components with different frequencies is 18 .

Therefore, the wind is decomposed by 18 layers and acquires eighteen IMF components. Here, we put the IMF components in the Narnet and the LSTM model, respectively, and each component was predicted by the model independently. Specifically, the first 310-day IMF components (44784 points) serve as the train set of two artificial intelligence algorithms above, and the 311-341 days of these components (4320 points) serve as the verification set of these models; then, the $342^{\text {nd }}$ component is the test set for observed values. To implement wind prediction at 144 steps (24 hours), the predicted values will input in the model after the first forecasting and computes 144 values iteratively. Ultimately, the linear superposition of prediction of all IMF components is the wind predicted result of the $342^{\text {nd }}$ day. For instability of the neural network, the whole iterative process mention needs to be repeated 10 times and averaged. The calculation result is presented in Figure 3.

Figure 3 presents that, before point A, the LSTM and the Narnet predicted the wind exact, and after point $A$, the predicted wind speed value of the LSTM model moves away from the observed value, and there is no overlap between the predicted and observed values basically. And after point B, the value of the Narnet deviates from the observed value; still, the predicted trend is consistent with the actual trend. To quantitatively evaluate the prediction accuracy of the Narnet and LSTM model, MAE and MAPE were computed in 24 hours, respectively. The calculation results are listed in Table 2.

Table 2 lists that MAE and MAPE of the Narnet are 1.1218 and $21.83 \%$, and the counterparts of LSTM are 3.9440 and $83.73 \%$, that is, 2.822 and $61.90 \%$ lower than that of the Narnet, respectively. In other words, MAE and MAPE values of LSTM prediction are above 3.000 and 50\% severally so that Narnet model's wind speed prediction results are more accurate than LSTMs. To analyze and compare the accuracy 


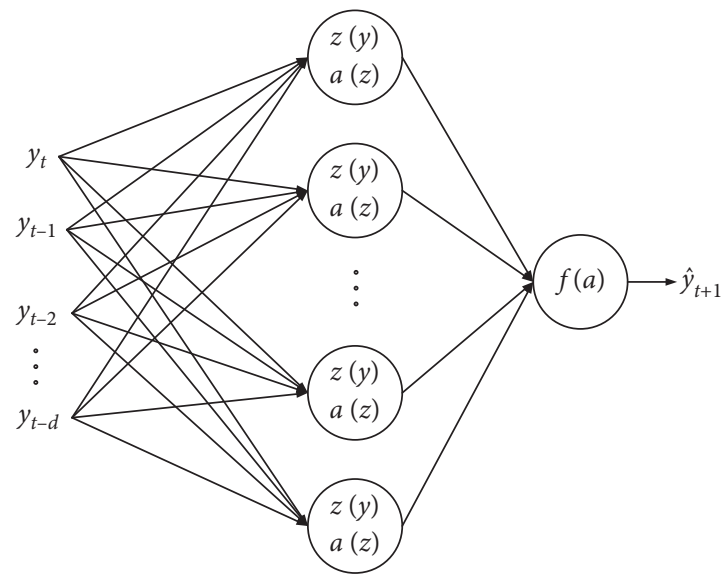

Figure 1: Topology of the Narnet model.

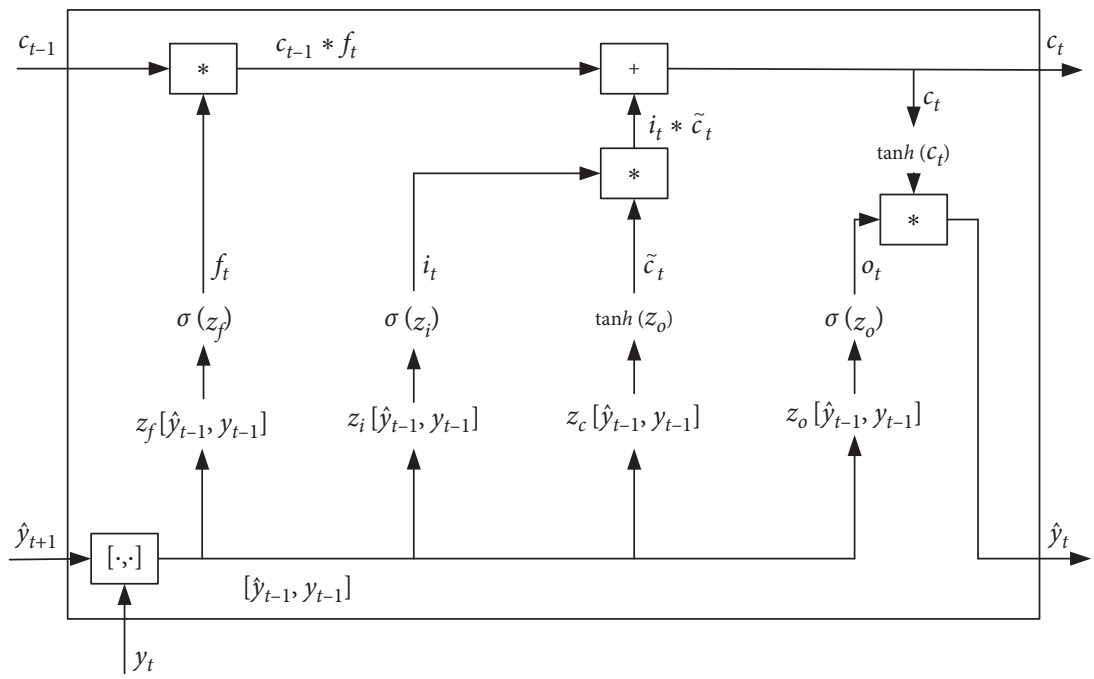

Figure 2: Topology of LSTM model.

variation in two models' prediction with step length further, we compute the absolute error between the forecast value and the observed value, and the result is shown in Figure 4.

As presented in Figure 4, at point A $(31,1.09777)$, the error of the LSTM model was more than $1 \mathrm{~m} / \mathrm{s}$ for the first time, and then, the error was raised, which means that the LSTM model predicted wind speed in acceptable 31 steps (about $5.2 \mathrm{~h}$ ). In contrast, the prediction of the Narnet model is acceptable in 72 steps (at point B $(72,-1.0951)$ ), for the error of the Narnet prediction was less than $1 \mathrm{~m} / \mathrm{s}$ in 72 steps (about $12 \mathrm{~h}$ ). Therefore, for the wind speed on the same day, the forecasting time horizon of the LSTM model wind speed is 41 steps (about 6.8 hours) narrower than the Narnet. The computed results demonstrated that the forecasting time horizon of the Narnet was wider than that of LSTM; however, the prediction was unacceptable after 72 steps. For improving the accuracy of prediction of the Narnet further in 24 hours, the length of predict steps was reduced to 72 . The wind speed rolling prediction results and prediction error are shown in Figure 5.
Figure 5 illustrates that Narnet model's predicted step size after 12 hours decreases to 35 steps compared to the previous (72 steps) in the wind speed prediction and the error beyond $1 \mathrm{~m} / \mathrm{s}$ at point $\mathrm{B}(107,1.36006)$ for the first time. MAE and MAPE values of prediction results $(0: 00$ and 12:00 (72 steps) and 12:00 and 24:00 (72 steps)) were calculated, respectively. The results are listed in Table 3.

As listed in Table 3, the MAE value of the Narnet model's prediction is 0.2849 in the 12 hours after $(00: 00$ to $12: 00)$, which is 0.5368 lower than after 12 hours $(0.8217)$, and the MAPE value is $4.79 \%$, which is $6.32 \%$ lower than after 12 hours $(11.11 \%)$. Despite the length of the forecast time horizon is the same, the trained model still gave different accuracies, which indicated that wind condition was variable between before and after noon. We characterized the variable by sample entropy, computed, and rolled the observed wind speed data in 12 hours (72 steps), and the results are depicted in Figure 6.

Figure 6 shows that the complexity of the observed wind speed in time has raised sharply after point A $(66,1.20397)$, 
TABLe 1: Central frequency $\left(\omega_{k}\right)$ of IMF at different $k$ values.

\begin{tabular}{lcccccccccc}
\hline$k$ & IMF 1 & IMF 2 & IMF 3 & $\ldots$ & IMF 16 & IMF 17 & IMF 18 & IMF 19 & IMF 20 & IMF 21 \\
\hline 2 & 5.55 & 606.58 & - & - & - & - & - & - & - & - \\
3 & 3.67 & 366.12 & 948.61 & - & - & - & - & - & - & - \\
$\ldots$ & $\ldots$ & $\ldots$ & $\ldots$ & - & - & - & - & - & - \\
16 & 0.47 & 78.32 & 204.12 & $\ldots$ & 4661.06 & - & - & - & - \\
17 & 0.47 & 78.20 & 203.67 & $\ldots$ & 4155.91 & 4712.45 & - & - & - \\
18 & 0.47 & 78.04 & 203.01 & $\ldots$ & 3688.31 & 4164.82 & 4713.35 & - & - \\
19 & 0.47 & 77.92 & 202.55 & $\ldots$ & 3652.16 & 4070.63 & 4499.30 & 4851.58 & - \\
20 & 0.47 & 77.85 & 202.32 & $\ldots$ & 3284.23 & 3674.42 & 4077.48 & 4493.20 & 4845.27 & - \\
21 & 0.47 & 77.77 & 202.07 & $\ldots$ & 2974.13 & 3299.55 & 3684.33 & 4083.22 & 4493.84 & 4844.38 \\
\hline
\end{tabular}

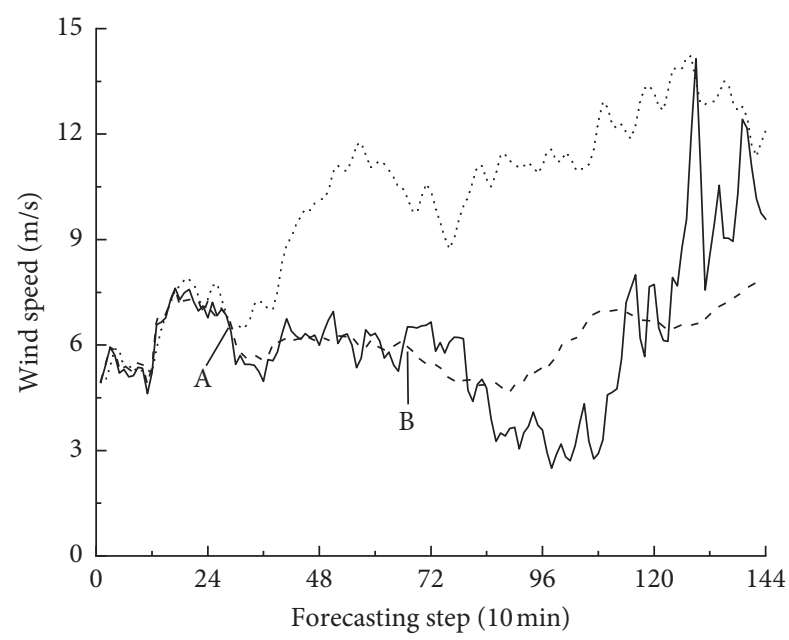

- Observed value
- - - Narnet-144-step ahead
$\ldots$.... LSTM-144-step ahead

Figure 3: Mean result of ten predictions.

TABLE 2: Evaluation of model prediction results.

\begin{tabular}{lcc}
\hline Model & MAE $(\mathrm{m} / \mathrm{s})$ & MAPE (\%) \\
\hline Narnet & 1.1218 & 21.83 \\
LSTM & 3.9440 & 83.73 \\
\hline
\end{tabular}

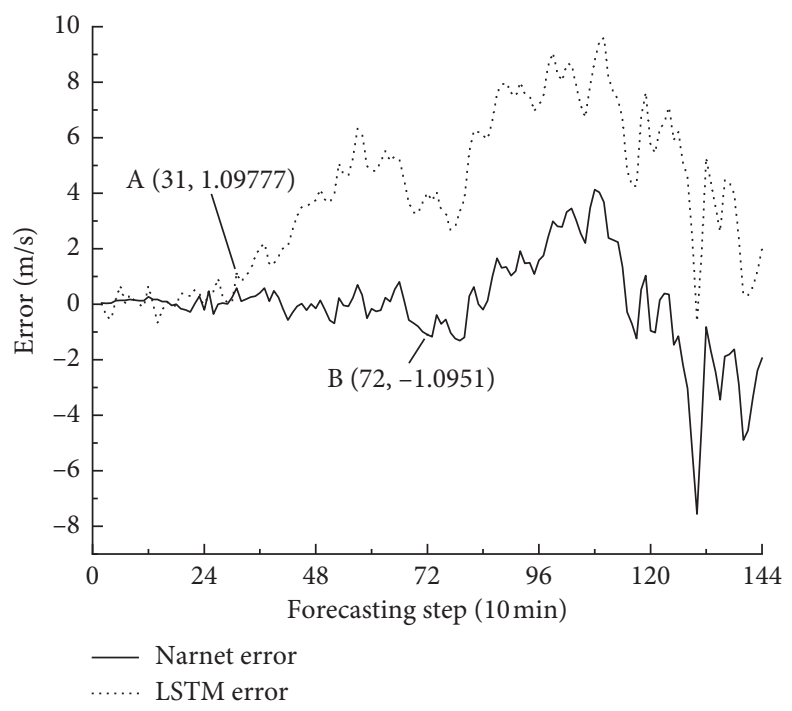

FIGURE 4: Error of model prediction results. 

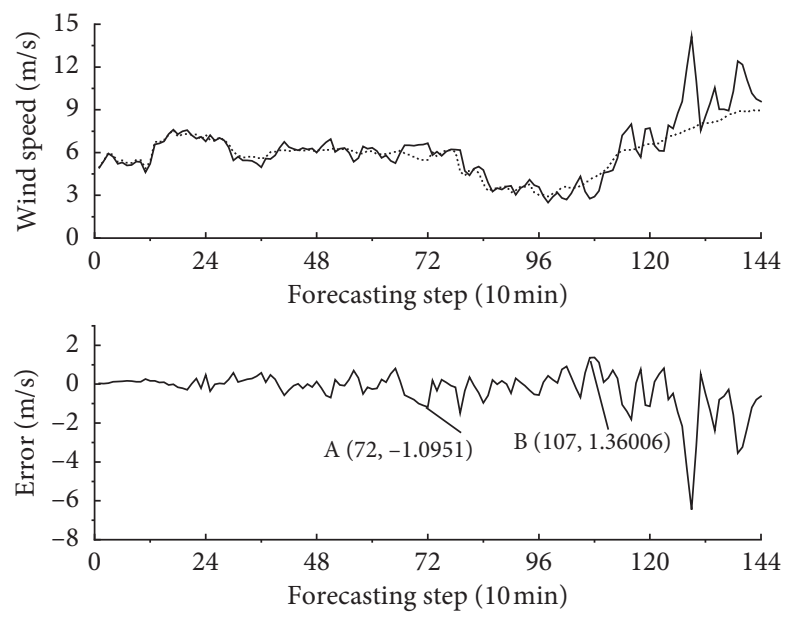

- Observed value

........ Narnet-72-step ahead

FIgURE 5: Verification result by Narnet.

TABLE 3: Evaluation of different periods by the Narnet.

\begin{tabular}{lcc}
\hline Time & MAE $(\mathrm{m} / \mathrm{s})$ & MAPE $(\%)$ \\
\hline $0: 00-12: 00$ & 0.2849 & 4.79 \\
$12: 00-24: 00$ & 0.8217 & 11.11 \\
\hline
\end{tabular}

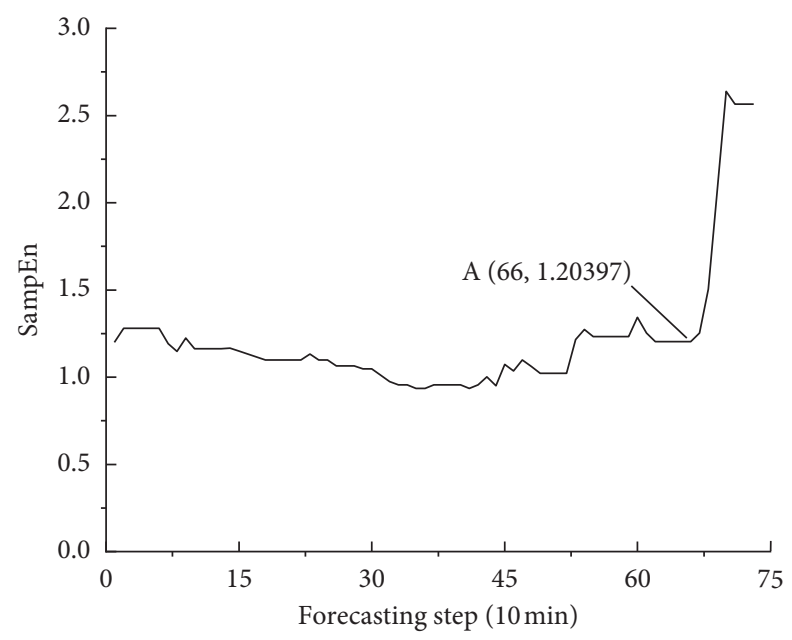

FIgURE 6: Rolling calculation of wind speed sample entropy.

the leap of sample entropy indicating that the complexity in time was different after the $66^{\text {th }}$ step $(11: 00-23: 00)$. Unfortunately, on account of the lack of relevant meteorological data, the reason for this leap was unknown. However, the low accuracy from the leap solved by adding times of prediction is effective. In fact, this is one of the reasons that the accuracy of prediction is better when the steps are shorter in most research literature. Specifically, the VMDNarnet hybrid method was adopted to compute the diurnal wind speed three times: $1 \mathrm{~h}-12 \mathrm{~h}$ (72 steps) was computed for the first time, $12 \mathrm{~h}-18 \mathrm{~h}$ (36 steps) was calculated for the second time, and $18 \mathrm{~h}-24 \mathrm{~h}$ (36 steps) wind speed was calculated for the third time. The three-time rolling prediction results of the Narnet model are depicted in Figure 7.

The leap of sample entropy shown in Figure 6 indicated that the law of wind speed is more complicated on the timeline and represented the credibility of prediction is reduced after the moment sample entropy leaped. Therefore, we corrected the prediction after point A by shortening the forecasting step and predicted again. As presented in Figure 7, the result of three times prediction approximates the observed wind speed. Here, we computed the MAE and MAPE values as 0.3476 and $6.31 \%$ and compared them to the one-time prediction of 144 steps; 


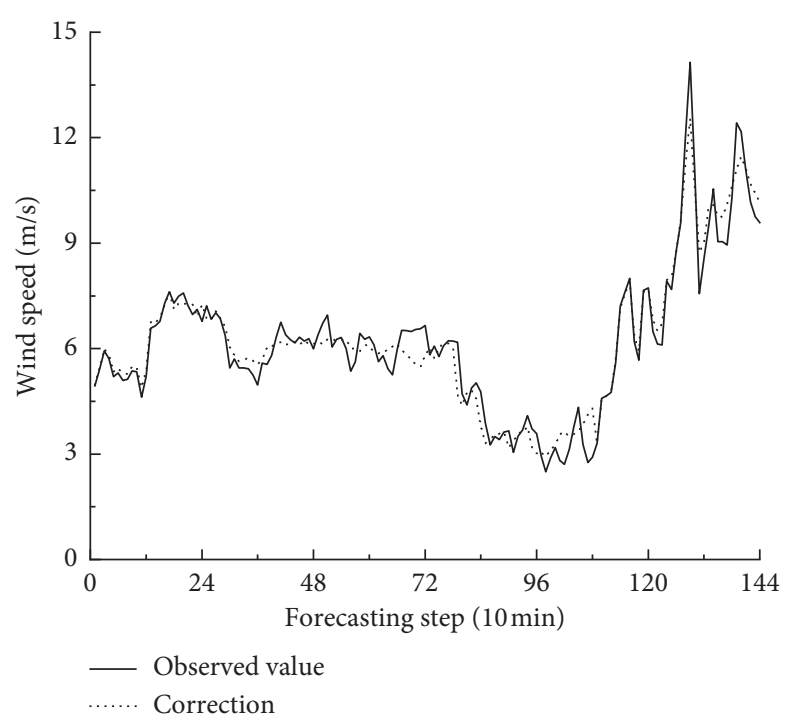

FIgURE 7: Correction of the Narnet model.

the counterpart of three times decreased by 0.7742 and $15.52 \%$, respectively. The prediction indicated that this correction is effective.

\section{Conclusions}

In this work, we proposed a diurnal wind speed forecast model, which provided a foundation for wind farm operation and maintenance strategies, thus achieving the goal of reducing wind power generation cost. The model mixed variational mode decomposition and nonlinear autoregressive network (VMD-Narnet) and was assessed by MAE and MAPE. The VMD-Narnet had superior performance than VMD-LSTM on the wind speed forecasting issue. We compared this method with the mixed model of variational mode decomposition and long-term and short-term memory network (VMD-LSTM), and the results showed that the values of MAE and MAPE of the VMD-Narnet hybrid method decreased by 2.8222 and $61.90 \%$. The wind speed complexity measured by sample entropy changed with time, and the leap of it led to inaccurate prediction results. Accordingly, the predictable step size of VMD-Narnet is 72 steps in the first 12 hours of the day and is 34 steps in the last 12 hours. Then, the accurate daily wind speed prediction results of VMDNarnet are obtained in three-time prediction.

In the next work, we will try to improve the Narnet model by deepening the number of topological layers and further improving the prediction accuracy of 24-hour wind speed. Also, the VMD-Narnet was demonstrated to be effective on the 24-hour wind speed forecasting; however, the meteorological reasons for sample entropy's leap were not explored. We will combine the actual records of the wind farm to explore this leap and improve the model according to them. Also, the operation and maintenance strategies from the forecasting before will be generated and applied to actual wind farms to drive costs of wind power down.

\section{Data Availability}

The data of wind speed used to support the findings of this work are currently under embargo while the research findings are commercialized. The data request of this work will be considered by the corresponding author at 18 months after publication of this article.

\section{Conflicts of Interest}

The authors declare that there are no conflicts of interest regarding the publication of this paper.

\section{Acknowledgments}

This work was supported by the National Natural Science Foundation of China (Grant no. 51965034), Lanzhou City Innovation Entrepreneurship Project (no. 2018-RC-25), and Chongqing Basic Science and Frontier Technology Research Special (no. CSTC2018jcyjAX0414). The authors would like to acknowledge the generous help from the School of $\mathrm{Me}$ chanical and Electronic Engineering and College of Automation.

\section{References}

[1] GWEC, Global Wind Statistics, https://www.compositesworld. com/news/gwec-reports-513-gw-of-new-wind-capacity-in-2018, 2018.

[2] A. Sharifian, M. J. Ghadi, S. Ghavidel, L. Li, and J. Zhang, "A new method based on type-2 fuzzy neural network for accurate wind power forecasting under uncertain data," Renewable Energy, vol. 120, pp. 220-230, 2018.

[3] J. L. Zhang, Y. M. Wei, and Z. F. Tan, "An adaptive hybrid model for short term wind speed forecasting," Energy, vol. 190, Article ID 115615, 2020.

[4] Z. R. Ma, H. W. Chen, J. J. Wang et al., “Application of hybrid model based on double decomposition, error correction and deep learning in short-term wind speed prediction," Energy 
Conversion and Management, vol. 205, Article ID 112345, 2020.

[5] Y. Q. Li, A. Bo, and H. T. Li, "Short-term wind speed prediction based on NARX and chaos-support vector machine," Power System Protection and Control, vol. 47, no. 32, pp. 65-73, 2019.

[6] C. Tian, Y. Hao, and J. Hu, "A novel wind speed forecasting system based on hybrid data preprocessing and multi-objective optimization," Applied Energy, vol. 231, pp. 301-319, 2018.

[7] S. Hanifi, X. L. Liu, Z. Lin et al., "A critical review of wind power forecasting methods-past, present and future," Energies, vol. 13, no. 15, Article ID 3764, 2020.

[8] H. Jahangir, M. A. Golkar, and F. Alhameli, "Short-term wind speed forecasting framework based on stacked denoising auto-encoders with rough ANN," Sustainable Energy Technologies and Assessments, vol. 38, Article ID 100601, 2020.

[9] S. Han, Y. P. Yang, and Y. Q. Liu, "Application study of three methods in wind speed prediction," Journal of North China Electric Power University, vol. 35, no. 3, pp. 57-61, 2008.

[10] K. Shi, F. Y. Wang, L. Chen et al., "A new wind speed prediction model based on random process considering autocorrelation," Power System Technology, vol. 41, pp. 529-535, 2017.

[11] Z. Y. Peng, S. Peng, L. D. Fu et al., "A novel deep learning ensemble model with data denoising for short-term wind speed forecasting," Energy Conversion and Management, vol. 207, Article ID 112524, 2020.

[12] Z. W. Niu, Z. Y. Yu, and W. H. Tang, "Wind power forecasting using attention-based gated recurrent unit network," Energy, vol. 196, Article ID 117081, 2020.

[13] C. S. Li, G. Tang, X. M. Xue et al., "Short-term wind speed interval prediction based on ensemble GRU model," IEEE Transactions on Sustainable Energy, vol. 11, no. 3, pp. 1370-1380, 2019.

[14] H. Liu, H. P. Wu, and Y. F. Li, "Multi-step wind speed forecasting model based on wavelet matching analysis and hybrid optimization framework," Sustainable Energy Technologies and Assessments, vol. 40, Article ID 100745, 2020.

[15] H. Liu, X. Mi, and Y. Li, "Comparison of two new intelligent wind speed forecasting approaches based on wavelet packet decomposition, complete ensemble empirical mode decomposition with adaptive noise and artificial neural networks," Energy Conversion and Management, vol. 155, pp. 188-200, 2018.

[16] Y. G. Zhang, G. F. Pan, B. Chen et al., "Short-term wind speed prediction model based on GA-ANN improved by VMD," Renewable Energy, vol. 156, pp. 1373-1388, 2019.

[17] Y. Zhang, B. Chen, G. Pan, and Y. Zhao, "A novel hybrid model based on VMD-WT and PCA-BP-RBF neural network for short-term wind speed forecasting," Energy Conversion and Management, vol. 195, pp. 180-197, 2019.

[18] Z. D. Tian, S. J. Li, Y. H. Wang et al., "Chaotic characteristics analysis and prediction for short-term wind speed time series," Acta Physica Sinica, vol. 64, no. 3, pp. 246-257, 2015.

[19] Q. Y. Yuan, C. Li, and Y. Yang, "Comparative study on chaos identification methods of wind speed time series," Journal of Engineering for Thermal Energy and Power, vol. 33, pp. 136-144, 2018.

[20] F. Zhang, D. S. Liu, J. C. Dai et al., "An operating condition recognition method of wind turbine based on SCADA parameter relations," Journal of Mechanical Engineering, vol. 55, no. 4, pp. 1-9, 2019.
[21] K. Dragomiretskiy and D. Zosso, "Variational mode decomposition," IEEE Transactions on Signal Processing, vol. 62, no. 3, pp. 531-544, 2014.

[22] Y. Y. Luo, Y. B. Yao, C. Huang et al., "Deformation feature extraction and analysis based on improved variational mode decomposition," Geomatics and Information Science of Wuhan University, vol. 45, no. 4, pp. 612-619, 2020.

[23] G. Benrhmach, K. Namir, A. Namir et al., "Nonlinear autoregressive neural network and extended Kalman filters for prediction of financial time series," Journal of Applied Mathematics, vol. 2020, Article ID 5057801, 6 pages, 2020.

[24] S. Hochreiter and J. Schmidhuber, "Long short-term memory," Neural Computation, vol. 9, no. 8, pp. 1735-1780, 1997.

[25] G. Memarzadeha and F. Keyniab, "A new short-term wind speed forecasting method based on fine-tuned LSTM neural network and optimal input sets," Energy Conversion and Management, vol. 213, Article ID 112824, 2020. 